\title{
Unilateral Alar Ulceration
}

Patrick S. Phelan, MD; Zachary P. Nahmias, MD; Shayna L. Gordon, MD; Caroline M. Mann, MD

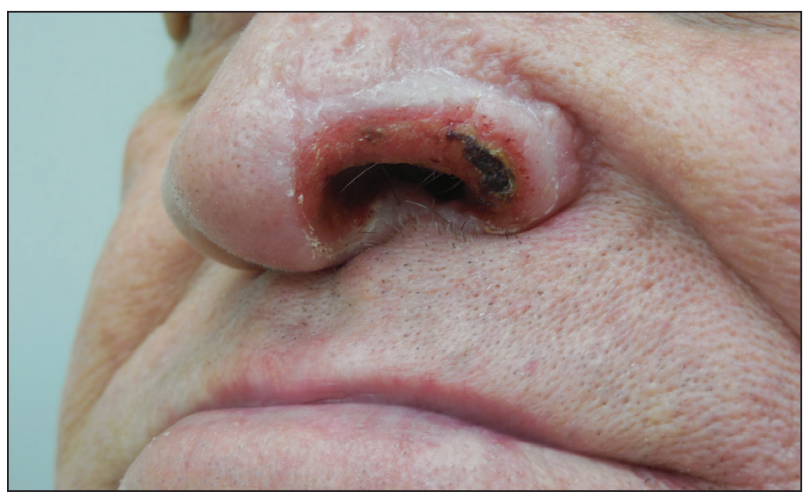

A 68-year-old man presented with a new left nasal alar ulcer following a recent episode of primary herpes zoster. Physical examination revealed erythema, erosion, and necrosis of the left naris with partial loss of the alar rim. Additional erythema was present without vesicles around the left eye and on the forehead.

\section{WHAT'S YOUR DIAGNOSIS?}

a. basal cell carcinoma

b. herpes simplex virus infection

c. mycobacterial infection

d. systemic vasculitis

e. trigeminal trophic syndrome

(self-induced trauma)

From the Washington University School of Medicine in St. Louis, Missouri. Drs. Nahmias, Gordon, and Mann are from the Division of Dermatology, Department of Medicine. 


\section{THE DIAGNOSIS:}

\section{Trigeminal Trophic Syndrome (Self-induced Trauma)}

$\mathrm{T}$ he patient admitted to manipulation of the ala in response to persistent pain despite resolution of the herpes zoster, for which he recently had completed a course of oral acyclovir. A preliminary diagnosis of trigeminal trophic syndrome (TTS) was made, and a subsequent punch biopsy revealed no evidence of malignancy. Topical antibiotic prophylaxis was prescribed, and he was instructed to avoid manipulation of the affected area. Treatment was initiated in consultation with pain specialists, and over the following 3 years our patient experienced a waxing and waning course of persistent pain complicated by new scalp and oral ulcers as well as alar impetigo. His condition eventually stabilized with tolerable pain on oral gabapentin and doxepin cream $5 \%$ applied up to 4 times daily. The alar lesion healed following sufficient abstinence from manipulation, leaving a crescent-shaped rim defect.

Trigeminal trophic syndrome classically is characterized by a triad of cutaneous anesthesia, paresthesia and/or pain, and ulceration secondary to pathology of trigeminal nerve sensory branches. Ulceration arises primarily through excoriation in response to paresthetic pruritus or pain. The differential diagnosis for TTS includes ulcerating cutaneous neoplasms (eg, basal cell carcinoma); mycobacterial, fungal, and viral infections (especially herpetic lesions); and cutaneous involvement of systemic vasculitides (eg, granulomatosis with polyangiitis). ${ }^{1}$ Biopsy is necessary to exclude malignancy, and ulcers may be scraped for viral diagnosis. Complete blood cell count and serologic testing also may help to exclude immunodeficiencies or disorders. Apart from viral neuropathy, common etiologies of TTS include iatrogenic trigeminal injury (eg, in ablation treatment for trigeminal neuralgia) and stroke (eg, lateral medullary syndrome).

\section{REFERENCE}

1. Khan AU, Khachemoune A. Trigeminal trophic syndrome: an updated review. Int J Dermatol. 2019;58:530-537. 